\title{
The Double Asteroid Redirection Test Mission
}

\author{
Brian Kantsiper, Andy Cheng, and Cheryl Reed \\ The Johns Hopkins University Applied Physics Laboratory \\ 11100 Johns Hopkins Rd \\ Laurel, MD 20723 \\ 443-778-4739 \\ Brian.kantsiper@jhuapl.edu
}

\begin{abstract}
On Feb. 15, 2013, a 20-meter asteroid unexpectedly hit the Earth without any warning near Chelyabinsk, Russia. This impact released about 500 kilotons TNT of energy, injured over 1500 people, and caused extensive property damage. The Chelyabinsk impact served as a dramatic reminder of the asteroid impact hazard and reemphasized the importance of discovering hazardous Near Earth Objects (NEOs) and learning how to mitigate the threat they pose.
\end{abstract}

Mitigation of a hazardous NEO can be accomplished by deflecting it so that it misses the Earth. Strategies to deflect an asteroid include impacting it with a spacecraft (a kinetic impactor), pulling it with the gravity of the mass of a spacecraft (a gravity tractor), using the blast of a nearby nuclear explosion, and modifying the surface or causing ablation by various means including lasers or particle beams. None of these approaches has been tested on a NEO. The AIDA mission is a proposed international collaboration to demonstrate kinetic deflection, the most mature technique for mitigating the impact hazard of a Near Earth Object (NEO).

AIDA consists of two mission elements, the NASA Double Asteroid Redirection Test (DART) mission and the ESA Asteroid Impact Mission (AIM). The main objectives of the DART mission, which includes the spacecraft kinetic impact and an Earth-based observing campaign, are to:

- Perform a full scale demonstration of the spacecraft kinetic impact technique for deflection of an asteroid, by targeting an object large enough to qualify as a Potentially Hazardous Asteroid;

- Measure the resulting asteroid deflection, by targeting the secondary member of a binary NEO and measuring the period change of the binary orbit;

- Understand the hypervelocity collision effects on an asteroid, including the long-term dynamics of impact ejecta; validate models for momentum transfer in asteroid impacts, inferring physical properties of the asteroid surface and sub-surface.

The DART target is the secondary member of the binary asteroid 65803 Didymos, with the impact scheduled to occur in September, 2022. The DART impact on the secondary member of the Didymos binary at $\sim 7 \mathrm{~km} / \mathrm{s}$ will alter the binary orbit period by at least 4 minutes, assuming a simple transfer of momentum to the target. The period change may be significantly greater, as the momentum transferred to the target asteroid may exceed the incident momentum of the U.S. Government work not protected by U.S. copyright kinetic impactor, possibly by a large factor. The AIM spacecraft will characterize the asteroid target and monitor results of the impact in situ at Didymos, but the period change can be determined accurately solely with ground-based observatories, an approach that is only feasible because of the choice of a binary system as target.

AIM is currently in Phase A. DART held its Mission Concept Review on May 21-22, 2015. This paper summarizes the DART mission concept and the path forward in Phase $A$.

\section{TABLE OF CONTENTS}

1. INTRODUCTION 1

2. Planetary DefenSE ........................................2

3. AIDA MISSION ........................................................2

4. DART DESCRIPTION ........................................3

5. SUMMARY ......................................................5

ACKNOWLEDGEMENTS ...........................................6

REFERENCES ........................................................6

BIOGRAPHY .....................................................6

1. INTRODUCTION

On Feb. 15, 2013, an exceptionally close approach to Earth by the small asteroid 2012 DA14 was eagerly awaited by astronomers around the world, but a different small asteroid unexpectedly impacted Earth over Chelyabinsk, Russia the same day without warning. The Chelyabinsk meteor released several hundred kilotons TNT of energy and injured over 1500 people. This dramatic reminder of the asteroid impact hazard re-emphasized the importance of discovering hazardous asteroids and learning how to mitigate the hazards. The Asteroid Impact and Deflection Assessment (AIDA) mission will be the first demonstration of a mitigation technique to protect the Earth from a potential asteroid impact, by performing a spacecraft kinetic impact on an asteroid to deflect it from its trajectory.

AIDA is an international collaboration between NASA and ESA, consisting of two independent but mutually supporting missions, one of which is the asteroid kinetic impactor and the other is the characterization spacecraft. These two missions are, respectively, the NASA Double Asteroid Redirection Test (DART) and the ESA Asteroid Impact Mission (AIM). The AIDA target will be the binary asteroid (65803) Didymos, with the deflection experiment to occur 
in October, 2022. The DART impact on the secondary member of the binary at $\sim 7 \mathrm{~km} / \mathrm{s}$ will alter the binary orbit period, which can be measured by Earth-based observatories. The AIM spacecraft will rendezvous with Didymos in advance of the DART impact to characterize the asteroid target, and AIM will monitor results of the DART impact in situ, to measure precisely the deflection resulting from the kinetic impact experiment.

AIDA will return fundamental new information on the mechanical response and impact cratering process at real asteroid scales, and consequently on the collisional evolution of asteroids with implications for planetary defense, human spaceflight, and near-Earth object science and resource utilization. AIDA will return unique information on an asteroid's strength, surface physical properties and internal structure. Supporting Earth-based optical and radar observations, numerical simulation studies and laboratory experiments will be an integral part of the AIDA mission.

\section{Planetary Defense}

Over the past couple of decades, the possibility of a Near Earth Object (NEO) colliding with the Earth has received increasing attention. The Chelyabinsk meteor fireburst has further heightened the public awareness of the risk such impacts can pose. The US Congress has assigned NASA the responsibility to characterize the threat posed by NEOs and to study concepts and to develop options to mitigate such threats [1]. NASA has now for many years been expanding our knowledge of the NEO population using both groundand space-based assets. As a result, the expected population of objects greater than $1 \mathrm{~km}$ in diameter is well surveyed [2], and existing sensors continue to look for smaller and smaller objects, with additional sensors coming on line and proposed [3].

Less has been accomplished to date in developing active mitigation options. A National Academies report divides possible active mitigation approaches into "slow push" and "slow pull" methods, kinetic impactors, and nuclear explosions [4], as shown in Figure 1. Of these, the kinetic impactor, in which the NEO is deflected by collision with a massive spacecraft at high relative velocity, is considered the most mature. The Deep Impact mission, although not designed to demonstrate deflection and targeting a comet rather than an asteroid (which represents the great majority of the threat population), did illustrate the feasibility of such an approach.

With the advances in the survey of the NEO population, NASA is prepared to turn to demonstration and prototyping of mitigation options. The kinetic impactor is the natural approach to begin with because of the maturity of the concept and the need to validate impact models if such a demonstration could be accommodated within the budgetary constraints.

\section{AIDA MISSION}

AIDA will be the first mission to demonstrate the kinetic impactor approach to NEO deflection. Ideally, such a demonstration would consist of two components, one spacecraft to function as the kinetic impactor and another in orbit around the target NEO to characterize the target before and after the impact. Previous attempts at such a project failed due to complexity and cost. $[5,6]$

The AIDA mission has two major differences from previous proposals to demonstrate kinetic deflection. The first is that it is an international collaboration. AIDA maintains the twocomponent approach of previous missions, but NASA provides DART, the kinetic impactor, while the European Space Agency (ESA) provides AIM, the NEO orbiter.

Such a collaboration frequently creates programmatic risk because of challenges aligning the schedule and organizational support for independently-managed projects. The second major difference is the choice of target. AIDA has selected the binary asteroid (65803) Didymos. Didymos has a close approach in 2022 in which it passes within 0.07 AU of Earth. Didymos is the only known NEO binary system that both makes such a close approach and can be reached with a reasonable $\Delta \mathrm{v}$ requirement, and the Didymos close approach does not recur for another $40 \mathrm{yr}$, as shown in Figure 2.

Targeting the secondary in the Didymos system (colloquially known as "Didymoon") during this close approach makes it possible to measure the deflection precisely using only ground-based measurements. The perturbation from the impact changes the orbit period very slightly, but, over time, this secular phase difference causes

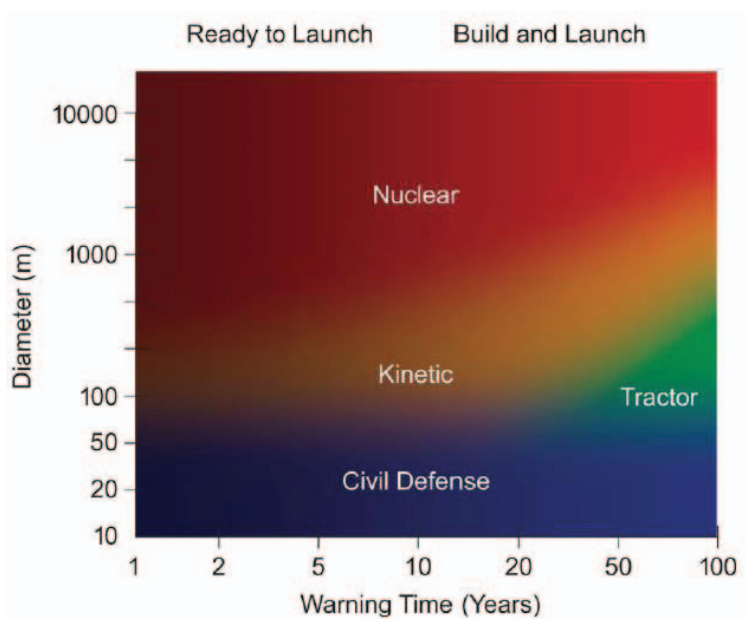

Figure 1 -- The preferred mitigation approach depends on the size of the NEO and the warning time before impact. [4] 
changes in the light curve that can be observed with groundbased assets. This provides a fallback to allow measurement of the deflection if, for any reason, AIM is unable to provide in situ measurement of the deflection.

Likewise, AIM is designed to maintain some programmatic independence from DART. Even in the absence of an impact, AIM would return interesting science relevant to planetary defense. Formation of binary NEO systems such as Didymos are thought to be driven by YORP effects [7], and AIM would be the first mission to such a system. AIM, with both an orbiting remote sensing package and a lander derived from the Rosetta MASCOT, would return valuable information on the system's dynamical state, geophysical properties, and structure. In addition, AIM includes a number of ESA technology demonstrations which are also independent of DART. [8]

\section{DART DESCRIPTION}

\section{Investigation}

The focus of the DART investigation is to measure the deflection achieved as a result of the impact. The net momentum change due to the impact can be greater than that of the impactor due to ejecta thrown off the target body as a result of the impact. The ratio of the momentum change achieved to the momentum of the impactor is known as $\beta$, and constraining this parameter for a real NEO in a relevant environment is a key goal of the DART investigation. It is critical in determining whether a kinetic impactor or set of impactors can be effective; the decision contour between nuclear and kinetic mitigations in Figure 1 depends critically on the expected value of $\beta$.

The achieved value of $\beta$ depends on a number of factors,

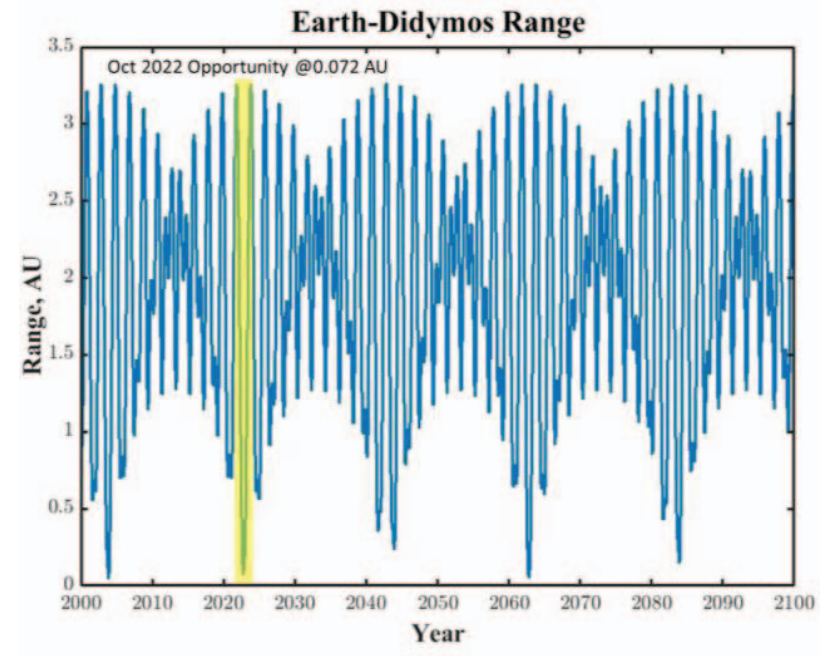

Figure 2 -- The close approach to Earth of the Didymos binary system that enables the AIDA mission concept does not occur again for $\mathbf{4 0}$ years. including the density, porosity, and effective strength of the target, the surface geometry relative to the impact, and, to a lesser extent, the properties and shape of the impactor itself. The dependency on the porosity is of particular interest, as asteroids of the "rubble pile" variety have porosity over a wide range of scale sizes, making it all but impossible to characterize the impact behavior in a laboratory setting. Preliminary models of the DART impact suggest a range of 1- 6 for $\beta$, depending on assumptions on the nature of the target body. [9] These models will continue to be improved over the course of the DART development, and the data returned from DART will be used to validate and improve these impact models.

This data from the mission will come from two sources, the DART spacecraft itself and ground-based assets. A wide range of ground-based telescopes will be used to capture light curves before and after impact. At impact, Didymos is expected to be near $14^{\text {th }}$ apparent magnitude, making it observable with even modest $(\sim 50 \mathrm{~cm})$ size telescopes with sort integration times. The expected deflection is large enough that the new period should be measurable within weeks after impact.

In addition to the ground-based observations, the DART spacecraft itself will be providing data. On approach, data from the imager (described below) will be streamed back to Earth. The final transmission prior to impact will provide better than $40-\mathrm{cm}$ resolution imaging of the impact site. This data will provide additional context to support the reconstruction of the event in the impact models.

With AIM, the return from the investigation is improved dramatically. Characterization of the target before the impact gives much better inputs for the hypervelocity impact modeling effort. The orbiter captures the size and shape of the crater, as well as data on the dust environment created by ejecta from the impact. This data allows validation and potentially significant improvements of the impact models, which will inform the decision-making process for any real threats that emerge in the future.

\section{Mission Design}

The DART trajectory design is constrained primarily by timing the impact at the time of the close approach by Didymos to Earth in 2022. This provides a number of trajectory options which do not drive the spacecraft design significantly.

The baseline trajectory is shown in Figure 3. DART launches on Dec 18, 2020 and cruises for 653 days before the impact. There are backup trajectories that launch 12 and 18 months later. The latter provides only a 3 -month cruise prior to impact, which would stress the operations timeline. The 2020 launch was selected to preserve a backup with a more reasonable operations cadence. Both the baseline and 
backup trajectories require a launch $\mathrm{C}_{3}$ of $6 \mathrm{~km}^{2} / \mathrm{s}^{2}$.

One interesting feature of the baseline trajectory is that there is an opportunity for a flyby of (3361) Orpheus for the first few days of the window. This flyby is of interest for a number of reasons. First, strictly from the perspective of the DART mission, it provides an opportunity to test out the approach concept of operations (CONOPS), including both the ground-based and on-board autonomous navigation. It is also of interest scientifically, as it provides data on an additional NEO. Finally, Orpheus is representative of the types of asteroids that might be visited on a future human expedition. It is, in fact, on the list of possible targets, although not one of the most likely. This opportunity only exists for the first 5 days of the launch window. After that, the $\Delta \mathrm{v}$ to return to the impact trajectory becomes prohibitive. The total $\Delta \mathrm{v}$ for the mission is $180 \mathrm{~m} / \mathrm{s}$.

Because of the emphasis on cost control, the smallest launch vehicles available to deliver the spacecraft to the required $\mathrm{C}_{3}$ are preferred. To date, DART has focused on two options, the Athena II and the Minotaur-C, both to be launched out of CCAFS. The Athena II can deliver approximately $300 \mathrm{~kg}$ to the DART departure trajectory, while the Minotaur C provides greater launch capacity.

Navigation follows a standard approach of handoff from radiometric to ground-based optical navigation (OpNav) to on-board autonomous navigation for the final approach. Two-way coherent Doppler and range is the primary approach for radiometric navigation during cruise and early approach, with occasional Delta Differential One-way Range (DDOR) near critical events.

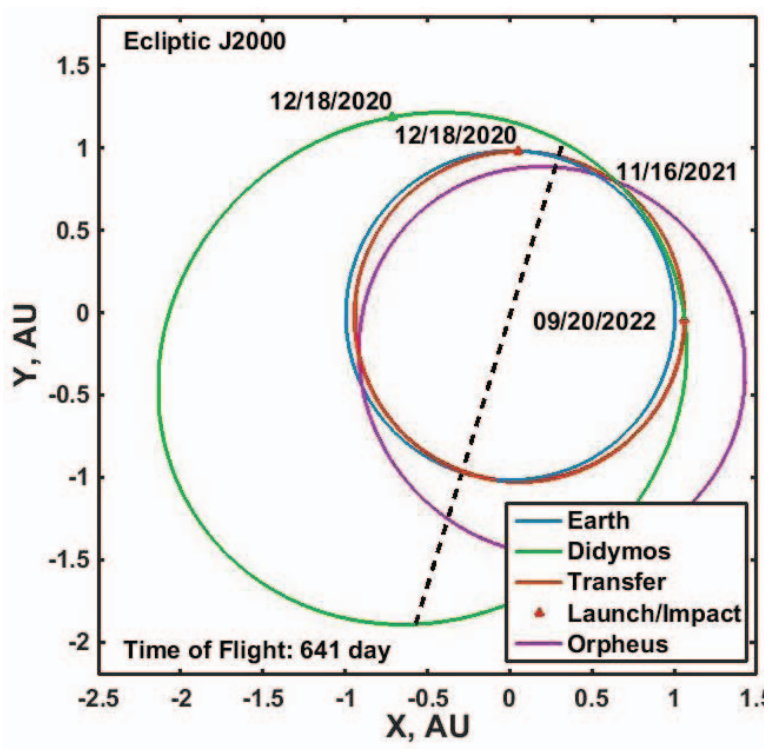

Figure 3 -- The DART baseline mission design is a low-energy trajectory with a long cruise and no deterministic maneuvers.
The on-board Didymos Reconnaissance and Asteroid Camera for OpNav (DRACO) can detect Didymos as far out as three months prior to impact to support OpNavs. At the handoff to the on-board autonomous navigation twelve hours prior to impact, the requirement is for $15 \mathrm{~km}(3-\sigma)$. The expected performance based on past missions is expected to be better.

Terminal guidance is implemented with the JHU/APL Small-body Maneuvering Autonomous Real-Time Navigation (SMARTNav) software. SMARTNav draws on JHU/APL's extensive experience in guidance for missile systems, as well as space applications such as the Midcourse Space Experiment (MSX) and work done for the Comet Nucleus Tour (CONTOUR). The target body is $170 \mathrm{~m}$ in diameter. Predicted performance is adequate to target this size body.

While Didymos is detectable well in advance of impact, the secondary cannot be resolved until much closer. DART targets the primary until the secondary is well-resolved. This occurs approximately $2 \mathrm{hr}$ prior to impact and is limited by the timing of the approach. The phase of the orbit that maximizes the orbital period change results in the secondary being in conjunction with the primary when the objects would otherwise resolve. The 2-hr timeline, however, provides more than enough opportunity to divert to the secondary.

There remains some uncertainty in the details of the orbit of the Didymos secondary. The impact of a $300 \mathrm{~kg}$ spacecraft is expected to change the velocity of the target by approximately $0.4 \mathrm{~mm} / \mathrm{s}$. As long as the impact occurs at a time where the orbit velocity is not nearly perpendicular to the approach velocity, this velocity change will result in a change in the orbital period. The DART impact is expected to change the period by $4.6 \mathrm{~min}$ (out of $\sim 12 \mathrm{hr}$ ). In less than three weeks, this period change would change the phase of the secondary by one quarter of the orbit, easily observable from the ground.

\section{Instrument}

The DRACO imager supports both terminal navigation and for characterization of the target in general and the impact site in particular. DRACO is an evolution of the Long-range Reconnaissance Imager (LORRI) on New Horizons which has provided the panchromatic images of the Pluto system. [10] DRACO includes minor modifications to incorporate a more modern focal plane array.

DRACO is a 20 -cm catadioptric Ritchey-Chrétien telescope. As discussed above, it supports OpNav as far out as three months prior to impact in a multi-second integration mode. LORRI has demonstrated a detection threshold of better than $17^{\text {th }}$ magnitude in this mode. [11] As Didymos approaches, the integration time must be reduced to avoid 
saturation. This actually helps in the terminal guidance, as the stars in the background are suppressed by the shorter integration time, simplifying the scene.

On final approach, DRACO takes images roughly every $5 \mathrm{~s}$. These images are streamed out at the maximum data rate. The 0.29 deg field of view subtends the entire target until seconds before impact and provides better than $40 \mathrm{~cm}$ resolution imaging of the body (with a goal of better than 20 $\mathrm{cm})$.

\section{Spacecraft}

The DART spacecraft is designed around the geometry of the final approach, as shown in Figure 4. On final approach, DRACO is pointed at the target. The spacecraft is rotated around the DRACO boresight to orient the high-gain antenna toward Earth. A single-axis gimbal is included to offset the slight variations from different launch dates within the launch window. The solar arrays are not articulated and are mounted so as to get near-full insolation in this attitude.

The spacecraft is three-axis controlled using thrusters only with a star tracker, IMU, and coarse sun sensors providing attitude information. The propulsion is a simple blowdown monopropellant system with one 22-N thruster for larger maneuvers and eight 1-N thrusters for attitude control, fine orbit corrections, and divert during terminal guidance.

In normal cruise and terminal approach, the solar arrays are fully insolated. The battery is sized to accommodate offpointing for trajectory change maneuvers. The avionics architecture is largely a rebuild of the Solar Probe Plus electronics currently in development. Communications are at X-band through the Deep Space Network. The HGA supports $3 \mathrm{Mbps}$ data rates for DRACO data return. A pair of low gain antennas provide low-rate communication in nearly any attitude.

The ground data system builds off of previous JHU/APL NASA missions. The only mission unique aspect of the ground system is an element that executes the SMARTNav algorithms in real-time with the DRACO data that is being transmitted. This real-time processing of the image data allows the operators to monitor SMARTNav performance. The 0.07 AU Earth range at impact corresponds to a roundtrip light time of just over a minute. At these ranges, the operators can monitor the SMARTNav performance, confirm the targeting algorithm correctly identifies the secondary, and perhaps take some limited corrective action if necessary.

\section{SUMMARY}

The DART Mission Concept Review (MCR) was held in May 2015. The project passed MCR and is funded to proceed in 2016. With such a straight-forward and simple implementation, the next phase is primarily focused on increasing the maturity of the design, there is one significant system-level trade that will be executed in this phase. It is possible that using electric propulsion to reach the departure

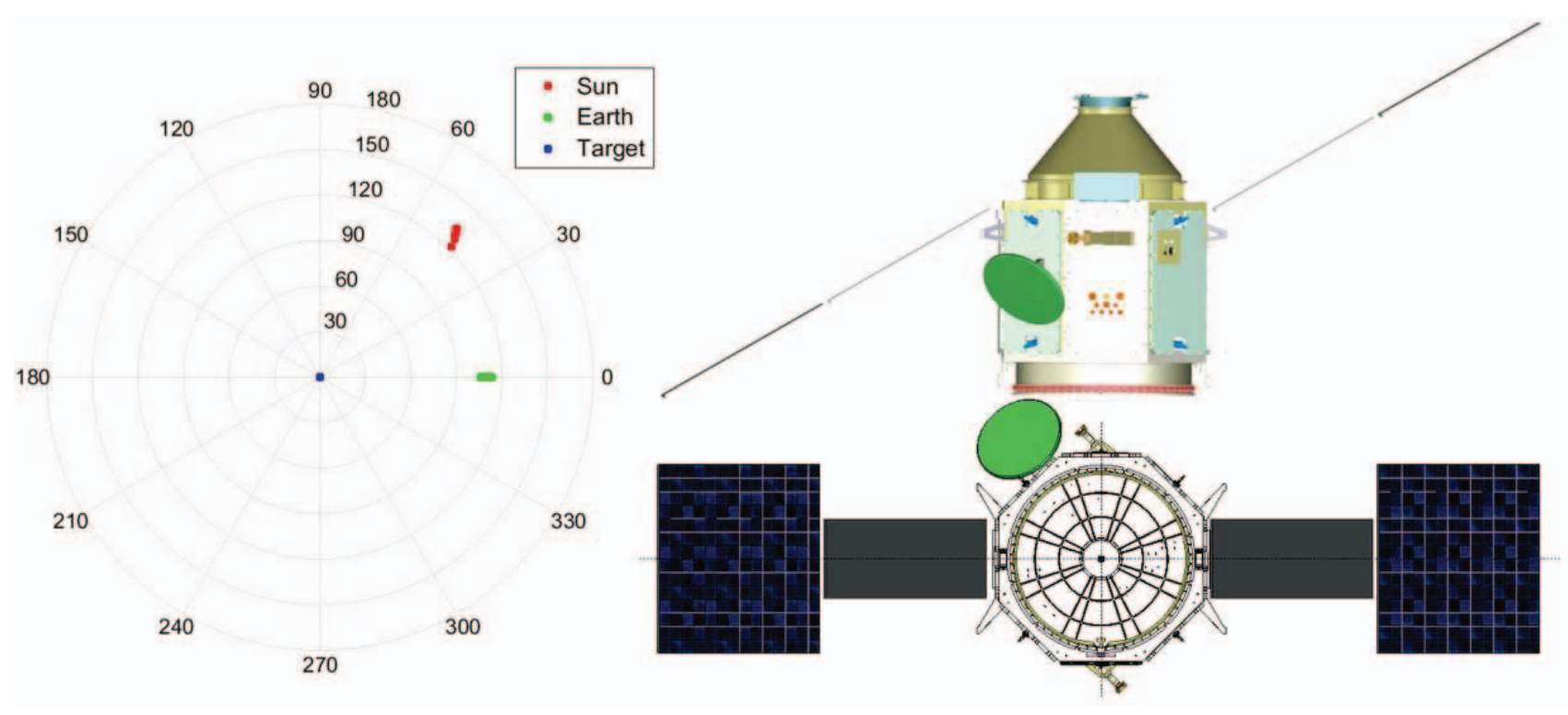

Figure 4 -- The DART spacecraft (right) is designed around the geometry of approach (left). The geometry shown illustrates the variations of the relative azimuth and elevation of the target, the Earth, and the Sun prior to final approach over the launch window. The sensor is pointed to the target, and the spacecraft rotates around the boresight to orient the high-gain antenna to Earth. The solar arrays are mounted to minimize cosine loss in this attitude. 
trajectory from the Earth system from a geosynchronous transfer orbit as a rideshare may be a more cost effective solution for the mission overall. After this trade is complete, the project will proceed toward a System Requirements Review in Aug 2016.

\section{ACKNOWLEDGEMENTS}

The authors gratefully acknowledge the support provided by the European Space Agency and the AIM team throughout the process of developing the AIDA mission and the DART concept.

\section{REFERENCES}

[1] Holdren, John P., Office of Science and Technology Policy, Letter to the Honorable John D. Rockefeller, IV and the Honorable Kay Bailey Hutchison. 15 Oct, 2010.

[2] Johnson, Lindley and Landis, Rob. Recent Enhancements to the NEO Observations Program: Implications for Planetary Defense. Planetary Defense Conference, Frascati, Italy, 13-17 April, 2015. IAA-PDC-15-01-04.

[3] Mainzer, A. et. al. Survey Simulations of a New NearEarth Asteroid Detection System. Astronomical Journal, 149 (5). Art. No. 172. ISSN 0004-6256.

[4] Defending Planet Earth: Near-Earth-object Surveys and Hazard Mitigation Strategies. Washington, D.C.: National Academies, 2010. 70-93. Print.

[5] "Past Studies: Don Quijote." http://www.esa.int/Our_Activities/Space Engineering_Te chnology/NEO/Past studies Don Quijote. European Space Agency. Web. 19 Oct. 2015.

[6] Chesley, S., et. al. ISIS Impactor for Surface and Interior Science. Meeting of the Small Bodies Assessment Group, Washington, DC, 10-11 July, 2013.

[7] Walsh, K.J., Richardson, D.C., Michel, P. Spin-up of rubble-pile asteroids: disruption, satellite formation, and equilibrium shapes. Icarus, 220, 514-529.

[8] "Asteroid Impact Mission (AIM)." http://www.esa.int/Our_Activities/Space_Engineering_Te chnology/NEO/Asteroid Impact Mission AIM. European Space Agency, 2 Apr. 2015. Web. 10 Oct. 2015.

[9] Stickle, A.M., Atchison, J.A., Barnouin, O.S., Cheng, A.F., Crawford, D.A., Ernst, C.M., Fletcher, Z. and A.S. Rivkin (2015) Modeling Momentum Transfer from Kinetic Impacts: Implications for Redirecting Asteroids, Procedia Engineering 103, 577-584. doi: 10.1016 /j.proeng.2015.04.075
[10] Stern, S. A., et. al., "The Pluto system: Initial results from its exploration by New Horizons," Science 16 October 2015: Vol. 350 no. 6258. DOI: 10.1126/science.aad 1815

[11] Cheng, A.F., et. al., "Long-Range Reconnaissance Imager on New Horizons," Space Sci.Rev.140:189215,2008. DOI: $\quad 10.1007 / \mathrm{s} 11214-007-9271-6$

\section{BIOGRAPHY}

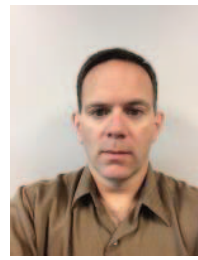

Brian Kantsiper is a Principal Professional Staff member at JHU/APL. $\mathrm{He}$ received an A.B. in Physics from Princeton University in 1992, and a Ph.D. in Aerospace Engineering from the Massachusetts Institute of Technology in 1998. He has been with the Johns Hopkins University Applied Physics Laboratory for 15 years. Prior to the AIDA mission, he was the mission system engineer on the Precision Tracking Space System. $\mathrm{He}$ is the assistant group supervisor of the Space Systems Engineering group in the Space Exploration Sector.

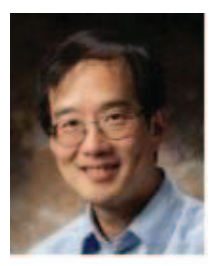

Andrew F. Cheng is a Principal Professional Staff member at JHU/APL. received an A.B. in Physics from Princeton University in 1971 and a Ph.D. in Physics from Columbia University in 1977. He is Chief Scientist for the Space Department at the Johns Hopkins Applied Physics laboratory. He was Deputy Chief Scientist for Space Science in NASA's Science Mission Directorate, at NASA Headquarters, from 2007 to 2008. He was a science team member for the Galileo, Cassini, Messenger, and Hayabusa missions. He was Project Scientist for the Near Earth Asteroid Rendezvous (NEAR) mission. He is currently Principal Investigator for the Long Range Reconnaissance Imager (LORRI) instrument on the New Horizons mission to Pluto and the Kuiper Belt.

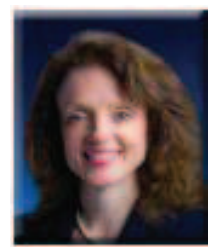

Cheryl Reed is a Principal Professional Staff member at JHU/APL, Head of Program Development for the Civilian Space (CS) business area, Deputy Program Manager for CS Exploration Programs, and the Standing Review Board Chair for NASA's GRAIL Mission. She served as Deputy Program Manager for the ORS Bus Standards Program and has been a management lead on numerous other NSS and CS projects since joining APL in 1985. Ms. Reed received an M.A. in international affairs from American University (1985) and a B.A. in business administration from New England College in Arundel, England (1982) 
